\title{
Brain scan diagnosis of central nervous system involvement in systemic lupus erythematosus
}

\author{
R. F. TAN, D. D. GLADMAN, M.B. UROWITZ, AND N. MILNE* \\ From the University of Toronto Rheumatic Disease Unit and the Nuclear Medicine Laboratory, Wellesley \\ Hospital, Toronto, Ontario, Canada
}

SUMmary Twenty-five patients with 29 episodes of active systemic lupus erythematosus with central nervous system involvement were studied according to a uniform protocol. Brain scans were found to be abnormal in all patients studied, and electroencephalograms were abnormal in $20 / 25$ patients. Serial brain scanning was found to be useful in the diagnosis of exacerbations and the monitoring of corticosteroid dosage.

In a recent study of 110 patients with systemic lupus erythematosus (SLE), we found major neuropsychiatric manifestations in 44 patients $(40 \%)$ including severe psychiatic disturbances or localised neurological lesions (Lee et al., 1977). The reported frequency of such manifestations in SLE ranges from 14-75\% (Bennahum and Messner, 1975; Feinglass et al., 1976), the incidence varying with the criteria used for inclusion.

In many instances, minor neuropsychiatric disturbances or severe headaches without localising signs are excluded because of poor neuropsychiatric and neuropathological correlations (Johnson and Richardson, 1968); yet these might represent active SLE with central nervous system (CNS) involvement. Available diagnostic procedures such as CSF examination or arteriography are frequently not helpful in diagnosing active CNS lupus.

Recent evidence seems to indicate that the radionuclide brain scan can be useful in the diagnosis of CNS involvement in SLE (Bennahum et al., 1974). The present study reports our experience with radionuclide brain scan in SLE.

\section{Material and methods}

\section{PATIENTS}

Twenty-five patients with active CNS SLE were studied. There were 21 females and 4 males, with a mean age of 34.9 years (range 21-75 years with only 3 patients over the age of 50) and a mean duration of SLE of 4.7 years. These patients were studied

\section{Accepted for publication October 10, 1977}

Correspondence to Dr M. B. Urowitz, 651A Rheumatic Disease Unit, Wellesley Hospital, 160 Wellesley Street East, Toronto, Ontario, Canada M4Y $1 \mathrm{~J} 3$

*Present address: 32322 Azores Drive, Laguna Niguel, California 92677, USA. prospectively over a 1 year period from November 1974 to November 1975. Twenty-two patients had 1 episode each of CNS involvement, 2 patients had 2, and 1 patient had 3 episodes. Active CNS SLE was defined as the presence of one or more of the following: psychosis, intractable headache, seizures, organic brain syndrome, cranial nerve palsies, or hemiparesis.

\section{CLINICAL EVALUATION}

Each patient with SLE was assessed clinically according to a uniform protocol (Lee et al., 1977). Laboratory tests performed included complete blood counts, Westergren sedimentation rate, liver function tests, urinalysis, renal function tests, Coombs's test, VDRL, protein electrophoresis, latex fixation test, LE prep, fluorescent antinuclear factor test using rat liver slices, DNA binding by the Farr technique using ${ }^{125} \mathrm{I}$, total haemolytic complement $\left(\mathrm{CH}_{50}\right), \mathrm{C} 3$ levels, EEG, and brain scan. A lumbar puncture was performed on each patient and the cerebrospinal fluid (CSF) studied for cell count, differential, glucose, total protein and IgG levels, and cultures.

\section{CONTROLS}

Four patients with SLE ( 3 female and 1 male), mean age 29.8 years, and disease duration of $7 \cdot 1$ years had clinically active disease without CNS involvement at the time of study and were used as controls. A further 4 females with a mean age of 36.5 years and disease duration of 4 years had inactive SLE, clinically and serologically were also used as controls. Thirty-two brain scans of patients with miscellaneous diagnoses such as headache, head injury, dizzy spells, epilepsy, cerebrovascular accidents were included as controls. 


\section{RADIONUCLIDE STUDIES}

Patients were premedicated with $400 \mathrm{mg}$ of potassium perchlorate 1 hour before the intravenous injection of 15 millicuries $(\mathrm{mCi})$ of sodium 99 pertechnetate. Static brain scintiscans were performed $2 \frac{1}{2}$ to 3 hours later using a Searle Instrumentation Phogamma III/HP gamma camera with a 4000 hole low energy parallel hole collimator. Anterior, posterior, right and left lateral views were recorded on Polaroid film.

Brain scans were performed on SLE patients with each presentation of active CNS involvement. Most patients were restudied after an interval of 10-21 days, usually after an adjustment in therapy had been made.

\section{BRAIN SCAN INTERPRETATIONS}

Brain scan abnormalities fell into 5 distinct patterns the first seen on the lateral view showed a localised? parietal extension of increased activity with $\overrightarrow{\mathrm{F}^{3}}$ 'saddle shaped' outline (Fig. 1). The following patterns were identified on anterior-posterior views (a) a generalised peripheral rim widening (Fig. 2) (b) a blurring and thickening of the peripheral rin of radionuclide uptake with inward extension of parasagittal activity producing the "draped curtainos pattern (Fig. 3), and (c) a localised pattern of increased activity on the anterior view indicating increased activity in the fronto-parasagittal are

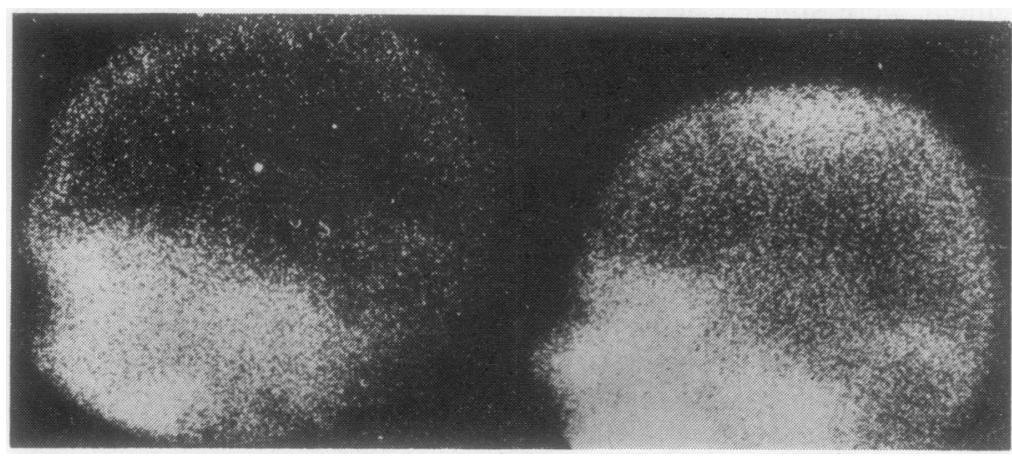

Fig. 1 Brain scan: left lateral view showing a localised parietal extension of increased radionuclide activity giving a 'saddle shaped' pattern. The normal left lateral view is shown on the left for comparison.

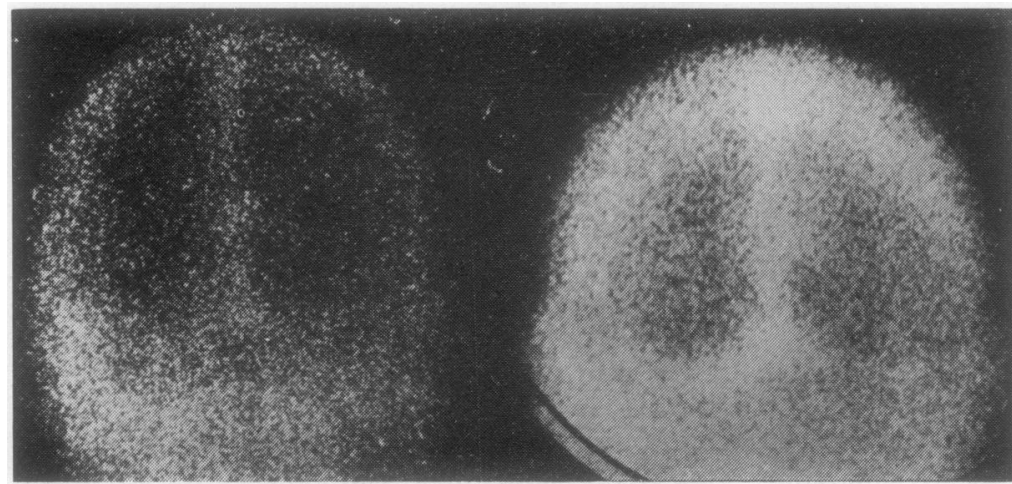

Fig. 2 Brain scan: posterior view showing a generalised 'peripheral rim' widening pattern. Normal posterior view on the left for comparison.

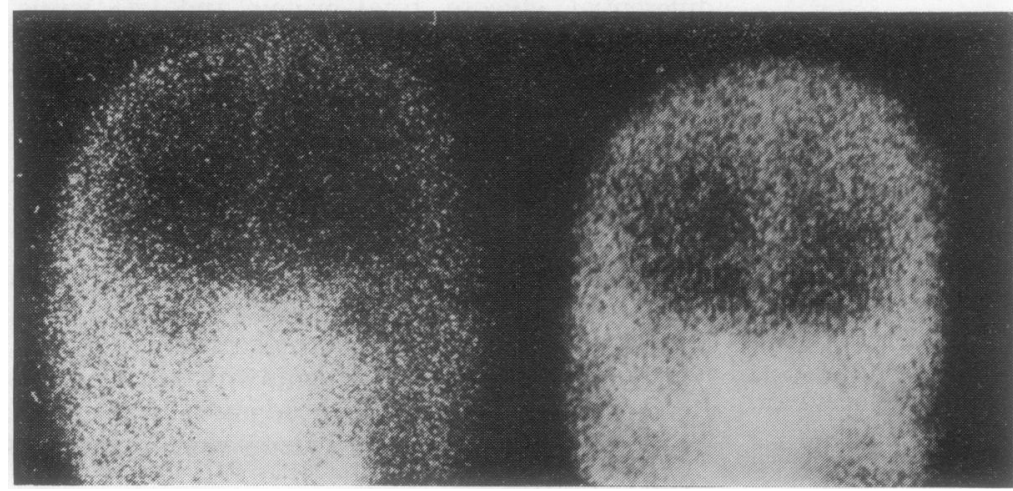

Fig. 3 Brain scan: anterior view showing $a$ 'draped curtain' pattern. Normal anterior view on left for comparison. 
(Fig. 4). The final pattern was a diffuse increase in radionuclide activity seen in all 4 views (Fig. 5 ). The common feature in all 5 patterns was their peripheral location. Reproducibility studies based on scans read blindly from the 25 patients and 32 controls revealed a reading accuracy of $96 \%$.

\section{THER A PY}

Once a brain scan abnormality compatible with CNS SLE was detected and CSF infection was excluded, prednisone therapy was either instituted or its dose increased. Prednisone dose was adjusted to control clinical symptoms and to achieve significant improvement toward eventual resolution of brain scan abnormalities.

\section{Results}

PATIENTS

The clinical features in the 25 patients with 29

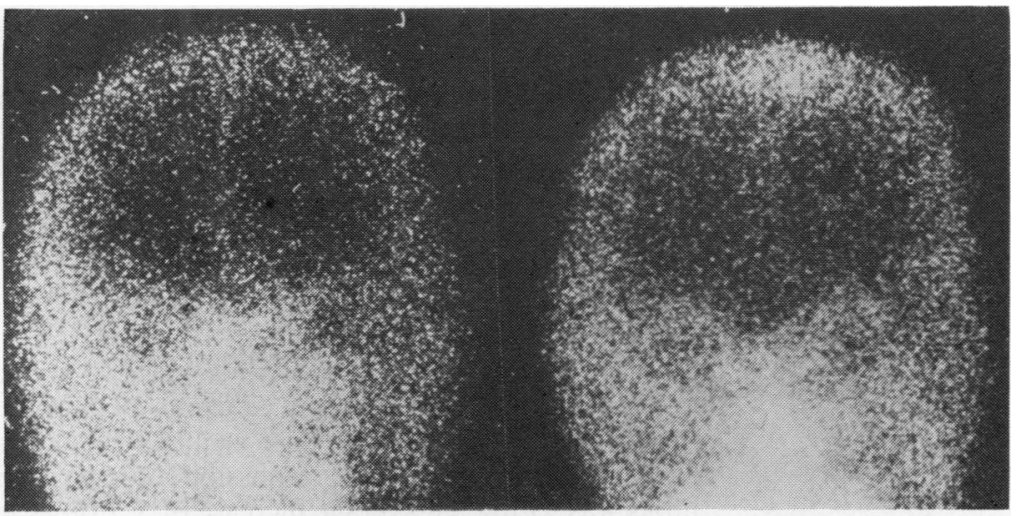

Fig. 4 Brain scan: anterior view showing a 'localised' pattern in the left frontoparasagittal area. Normal anterior view on left for comparison.

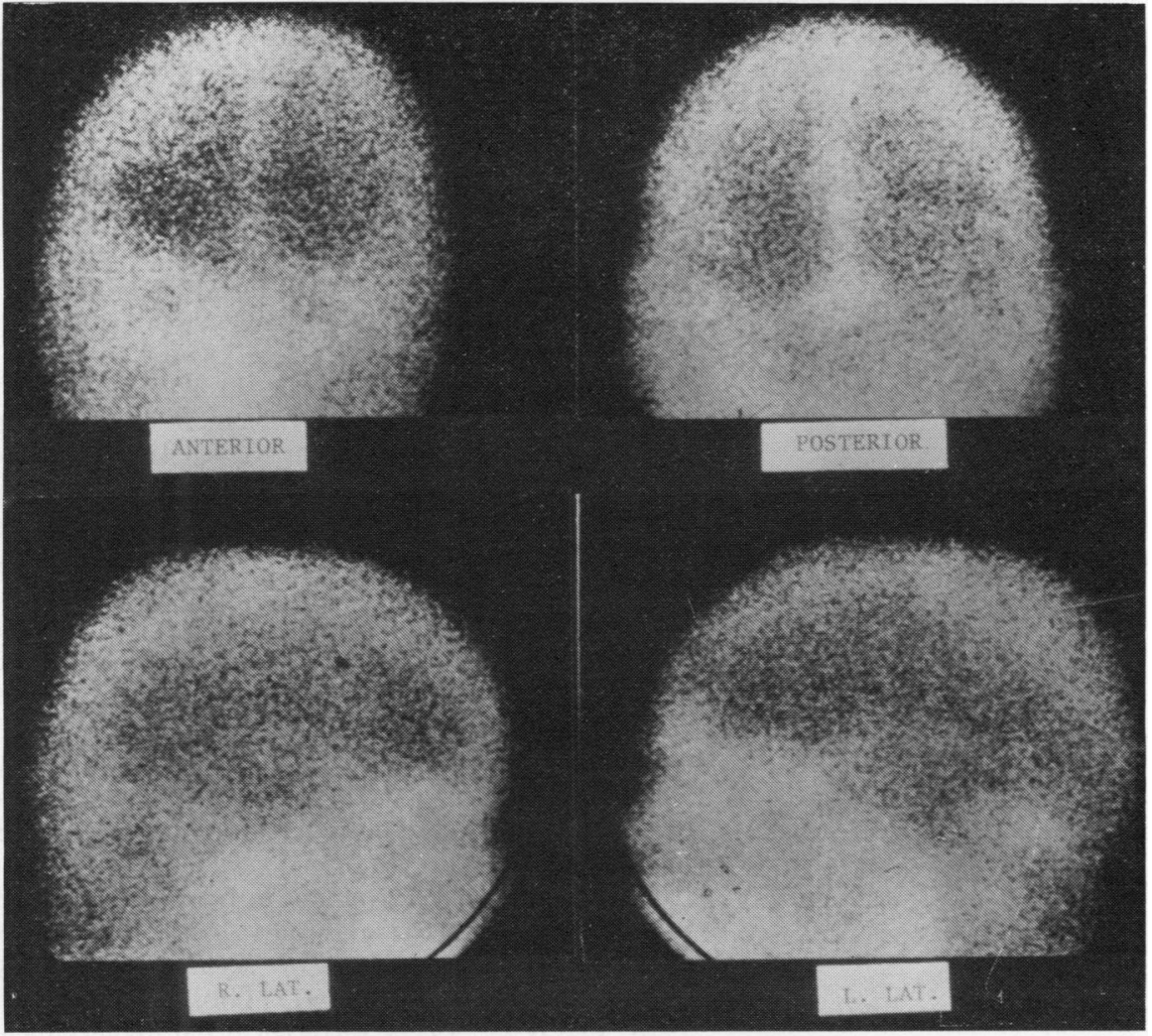

Fig. 5 Brain scan: anterior, posterior, and lateral views showing 'diffuse' pattern. 
episodes of CNS lupus are summarised in Table 1. As can be seen, 24 episodes of CNS lupus presented with psychosis. Twenty-one episodes were characterised by intractable headache persisting over 1 week and unresponsive to large doses of codeine. It is worth noting that in 6 patients the intractable headache was the sole presenting feature of their CNS lupus. In the remaining patients $(15 / 21)$ psychosis and intractable headache occurred simultaneously. Four vessel cerebral arteriograms were performed in 2 patients with seizures within 48 hours of hospitalisation and these were normal. Five patients had organic brain syndrome characterised by delirium, emotional inadequacy, loss of contact with reality, and impairment in concentration and/or memory as manifestations of CNS lupus which responded to corticosteroid therapy. One patient had-a transient partial left oculomotor nerve palsy. Both patients with hemiparesis responded completely to an increase in corticosteroid therapy.

\section{SEROLOGICAL EVALUATION}

The serological findings in the 25 patients are summarised in Table 2. ANF was detected in 25 of the 29 episodes and the LE prep was positive in 12 . Increased anti-DNA levels ( $>20 \%$ DNA binding by the Farr technique) were present in 18. Twentytwo of the 29 episodes had decreased levels of complement $\left(\mathrm{CH}_{50}\right)$ or $\mathbf{C} 3$.

\section{CNS EVALUATION}

The results of CNS investigation at the time of diagnosis are summarised in Table 3 . Of the 20 patients tested, none had CSF pleocytosis and only $50 \%$ had elevated CSF protein. Of the 13 patients tested, none had decreased CSF IgG levels. EEG was abnormal in 20 of the 25 patients tested. The most frequent (16/25) abnormalities on EEG were

Table 1 Clinical features of SLE CNS disease

\begin{tabular}{lc}
\hline Features & No. of episodes \\
\hline Psychosis & 24 \\
Intractable headache & 21 \\
Seizure & 6 \\
Organic brain syndrome & 5 \\
Cranial nerve palsy & 1 \\
Hemiparesis & 2 \\
\hline
\end{tabular}

Table 2 Serology in 29 episodes of SLE CNS disease

\begin{tabular}{ll}
\hline Test & No. abnormal \\
\hline ANF & 25 \\
LE prep & 12 \\
Anti-DNA Ab & 18 \\
Low CH CH $_{50}$ & 13 \\
Low $\mathrm{B}_{1} \mathrm{C}_{(\mathrm{C} 3)}$ & 21 \\
Low $\mathrm{CH}_{50}$ or $\mathrm{B}_{1} \mathrm{C}$ & 22 \\
\hline
\end{tabular}

Table 3 CNS investigation in 29 episodes of SLE CNS disease

\begin{tabular}{|c|c|c|}
\hline Test & No. tested & No. abnormal \\
\hline CSF cell count & 20 & $\mathbf{0}$ \\
\hline CSF protein & 20 & 10 \\
\hline CSF IgG & 13 & 0 \\
\hline EEG & 25 & 20 \\
\hline Brain scan & 29 & 29 \\
\hline
\end{tabular}

focal changes involving the temporal, frontøtemporal, or temporoparietal areas. Four patien had diffuse slow delta wave activity compatible with encephalopathy. None of these 4 patients had angy other underlying cause for encephalopathy. Brain scans were abnormal in all cases with suspected CNS lupus. No patients were found to have CNS infection. All brain scans performed on contriel lupus patients were normal.

\section{RESPONSE TO THERAPY}

As a group the mean daily prednisone dose at the time of diagnosis was $15.6 \mathrm{mg}$ (range 0-50 mg) add the mean daily prednisone dose required to obtin a therapeutic response or remission of the CISS lupus was $57 \mathrm{mg}$, an average increase of 3.7 times $\overrightarrow{\overrightarrow{\mathrm{fn}}}$ prednisone dosage.

Twenty-three of 25 patients improved on the and in these brain scans resolved toward nowial. Two patients died with active CNS lupus, despite wn increase in the prednisone therapy. Neuropathological examination in one was normal and in second revealed a sterile leptomeningitis wth evidence of cerebral atrophy and positive immunofluorescent staining for IgM in the choroid plexus.

\section{Discussion}

Central nervous system involvement in SLE common, yet remains difficult to diagnose in ma instances. This is especially true when CNS lupis presents as either a psychiatric disorder or $a s$ intractable headaches. Previous large series (Harvey et al., 1954; Estes and Christian, 1971; Duboris, 1974; Sergent et al., 1975; Feinglass et al., 1976) $\mathrm{Bf}$ f patients with CNS SLE, failed to emphasise importance of intractable headache as a feature Jof CNS involvement. In our series, 21 patients hid intractable headache as an important presentiog feature, Six of these patients had intractable headache as the sole presenting feature of their CAS lupus.

Although CNS involvement in patients with $S$ has been associated with more severe diseases as evidenced by clinical and/or serological activify (Bennahum and Messner, 1975; Gibson and Myeuts, 1976; Lee et al., 1977), serological parameters hợ 
been poor indicators of CNS involvement. Depressed serum complement and elevated DNA-antibodies may occur in patients with CNS SLE, but are not as consistent as in lupus nephritis, to be useful for diagnosis. As seen in Table 2, elevated DNAantibodies were detected in only 18 of 29 episodes and depressed $\mathrm{CH}_{50}$ or $\mathrm{C} 3$ complement levels were detected during 22 episodes of CNS lupus. CSF findings of increased DNA-antibodies, DNA-antiDNA complexes, reduced levels of $\mathrm{C} 3$ and IgG again are not consistent markers of active CNS disease (Petz et al., 1971; Levin et al., 1972; Hadler et al., 1973; Lindstrom and Sjoholm, 1975; Small et al., 1977).

The EEG was abnormal in $80 \%$ of our cases, but $5 / 25$ or $20 \%$ with active CNS involvement showed no abnormality. This finding is in agreement with that of both Feinglass et al. (1976) where $71 \%$ of those patients with CNS lupus had abnormal EEG findings, and Gibson and Myers (1976) who demonstrated abnormal EEG in $84 \%$ of patients with CNS SLE.

All of our patients with active CNS lupus had abnormal brain scans at the time of diagnosis of their disease. This is in agreement with Bennahum et al. (1974) who reported abnormal brain scans in 11/12 scans in 6 patients with active CNS lupus. It is, however, in marked contrast to other published reports. Feinglass et al. (1976) found only $2 / 26$ brain scans to be abnormal among 26 neuropsychiatric episodes, despite the fact that 20 of 24 patients with normal scans had focal neurological signs and/or seizures.

Only 4 of the patients studied by Gibson and Myers (1976) had abnormal brain scans. However, they did not state how the scans were performed and how many of their patients had scans. Small et al. (1977) detected only 2 abnormal static brain scans of 10 scans performed on patients with CNS SLE. Fifty per cent of flow studies performed on these patients were abnormal. Again the scanning method was not reported. The discrepancy between these reports and our results may in part be due to the different technique used in brain scanning. We used the gamma camera to record the brain scans whereas Feinglass et al. (1976) and perhaps the others (Gibson and Myers, 1976; Small et al., 1977) used the rectilinear brain scanner.

The rectilinear scanner accepts optimal radiation from a focal plane $2 \frac{1}{2}$ inches below the scalp, parallel to the collimator movement, but radiation from off focus planes is blurred. The gamma camera on the other hand, with the focal parallel hole collimator does not have a focal plane but accepts radiation from all over the scalp and may therefore be better for detecting pathology in the cortex.
We found sequential brain scans to be a reliable measure of either continued activity or resolution of CNS involvement, and relied on them in making therapeutic decisions. With definite deterioration in the clinical status associated with worsening of brain scan abnormalities in our patients we increased prednisone usually by doubling or tripling the previous dose until a clinical response or remission was attained.

Twenty-seven episodes of CNS lupus responded to therapy, 2 did not. Those patients in whom the brain scan abnormalities persisted or worsened were those who had a protracted clinical course of CNS lupus. They also required a higher dose of corticosteroid for a prolonged period of time for control of their CNS lupus. Two patients died with active CNS SLE despite high daily dose of corticosteroid (prednisone $100 \mathrm{mg}$ ). Neuropathological examination in one was normal and in the second revealed a sterile leptomeningitis with evidence of cerebral atrophy and positive immunofluorescent staining for IgM in the choroid plexus. This finding may support the hypothesis that the pathogenesis of CNS lupus may be secondary to an immune complex disease (Atkins et al., 1972).

Supported by a grant from the Canadian Arthritis Society no. 1-144-(70).

\section{References}

Atkins, C. I., Kondon, J. J., Quismorio, F. P., and Friou, G. J. (1972). The choroid plexus in systemic lupus erythematosus. Annals of Internal Medicine, 76, 65-72.

Bennahum, D. A., and Messner, R. P. (1975). Recent observations on central nervous system lupus erythematosus. Seminars in Arthritis and Rheumatism, 4, 253-266.

Bennahum, D. A., Messner, R. P., and Shoop, J. D. (1974). Brain scan findings in central nervous system involvement by lupus erythematosus. Annals of Internal Medicine, 81, 763-765.

Dubois, E. L. (1974). Lupus Erythematosus, 2nd ed., p. 308. University of Southern California Press, Los Angeles.

Estes, D., and Christian, C. L. (1971). The natural history of systemic lupus erythematosus by prospective analysis. Medicine, 50, 85-95.

Feinglass, E. J., Arnett, F. C., Dorsch, C. A., Zizic, T. M., and Stevens, M. B. (1976). Neuropsychiatric manifestations of systemic lupus erythematosus: diagnosis, clinical spectrum, and relationship to other features of the disease. Medicine, 55, 323-339.

Gibson, T., and Myers, A. R. (1976). Nervous system involvement in systemic lupus erythematosus. Annals of the Rheumatic Diseases, 35, 398-406.

Hadler, N. M., Gerwin, R. D., Frank, M. M., Whitaker, J. N., Baker, M., and Decker, J. L., (1973). The fourth component of complement in the cerebrospinal fluid in systemic lupus erythematosus. Arthritis and Rheumatism, 16, 507-521.

Harvey, A. M., Shulman, L. E., Tumulty, P., Conley, C. L., and Schoenrich, E. H. (1954). Systemic lupus erythematosus: review of the literature and clinical analysis of 138 cases. Medicine, 33, 291-437. 
Johnson, R. T., and Richardson, E. P. (1968). The neurological manifestations of systemic lupus erythematosus: a clinical-pathological study of 24 cases and review of the literature. Medicine, 47, 337-369.

Lee, P., Urowitz, M. B., Bookman, A. A. M., Koehler, B. E., Smythe, H. A., Gordon, D. A., and Ogryzlo, M. A. (1977). Systemic lupus erythematosus: a review of 110 cases with particular reference to lupus nephritis, CNS manifestations, infections, aseptic necrosis and prognosis. Quarterly Journal of Medicine, 46, 1-32.

Levin, A. S., Fudenberg, H. H., Petz, L. D., and Sharp, G. C. (1972). IgG levels in cerebrospinal fluid of patients with central nervous system manifestations of systemic lupus erythematosus. Clinical Immunology and Immunopathology, 1, 1-5.
Lindstrom, F. D., and Sjoholm, A. G. (1975). Cerebrospi fluid changes in systemic lupus erythematosus with and without central nervous system involvement. Arthritis and Rheumatism, 18, 413-414.

Petz, L. D., Sharp, G. C., Cooper, N. R., and Irvin, W.尹 (1971). Serum and cerebral spinal fluid complement afd serum autoantibodies in systemic lupus erythematosws. Medicine, 50, 259-275.

Sergent, J. S., Lockshin, M. D., Klempner, M. S., aํㅐㅇ Lipsky, B. A. (1975). Central nervous system disease $\overline{\text { In }}$ systemic lupus erythematosus: therapy and prognoss. American Journal of Medicine, 58, 644-654.

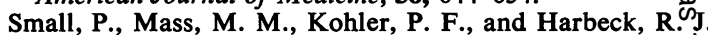
(1977). Central nervous system involvement in SI. Diagnostic profile and clinical features. Arthritis and Rheumatism, 20, 869-878. 